\title{
AR models of singular spectral matrices
}

\author{
Brian D.O. Anderson, Life Fellow, IEEE, Manfred Deistler, Fellow, IEEE Weitian Chen, Member, IEEE \\ and Alexander Filler
}

\begin{abstract}
This paper deals with autoregressive models of singular spectra. The starting point is the assumption that there is available a transfer function matrix $W(q)$ expressible in the form $D^{-1}(q) B$ for some tall constant matrix $B$ of full column rank and with the determinantal zeros of $D(q)$ all stable. It is shown that, even if this matrix fraction representation of $W(q)$ is not coprime, $W(q)$ has a coprime matrix fraction description of the form $\tilde{D}^{-1}(q)\left[I_{m} 0\right]^{T}$. It is also shown how to characterize the equivalence class of all autoregressive matrix fraction descriptions of $W(q)$, and how canonical representatives can be obtained. A canonical representative can be obtained with a minimal set of row degrees for the submatrix of $\tilde{D}(q)$ obtained by deleting the first $m$ rows. The paper also considers singular autoregressive descriptions of nested sequences of $W_{p}(q), p=$ $p_{0}, p_{0}+1, \ldots$, where $p$ denotes the number of rows, and shows that these canonical descriptions are nested, and contain a number of parameters growing linearly with $p$.
\end{abstract}

\section{THE PROBLEM OF INTEREST}

We consider stable autoregressive systems

$$
D(q) y_{t}=\nu_{t}
$$

Here $q$ is a complex variable as well as the backward shift on the integers,

$$
D(q)=\sum_{j=0}^{k} D_{j} q^{j}, D_{j} \in \mathbb{R}^{p \times p}
$$

the determinant of $D(q)$ satisfies the stability condition

$$
|D(q)| \neq 0,|q| \leq 1
$$

and $\left(\nu_{t}\right)$ is white noise. While engineers typically regard discrete-time transfer functions of finite-dimensional systems as best desribed using rational expressions in a variable $z$, a forward-shift operator, it is usually more convenient in

This work has been supported by ARC Discovery Project Grant DP0664427.

Support by the FWF (Austrian Science Fund) under contracts P17378 and P20833/N18 and by the Oesterreichische Forschungsgemeinschaft is gratefully acknowledged.

B.D.O. Anderson is with the Research School of Information Sciences and Engineering, Australian National University, Canberra, ACT 0200, Australia and Canberra Research Laboratory, National ICT Australia Ltd., PO Box 8001, Canberra, ACT 2601, Australia e-mail: brian.anderson@anu.edu.au

M. Deistler is with the Department of Mathematical Methods in Economics, Technical University of Vienna, 8/119 Argentinierstrasse, A 1040 Vienna, Austria. e-mail: deistler@tuwien.ac.at

W. Chen is with the Research School of Information Sciences and Engineering, Australian National University, Canberra, ACT 0200, Australia and Canberra Research Laboratory, National ICT Australia Ltd., PO Box 8001, Canberra, ACT 2601, Australia e-mail: weitian.chen@anu.edu.au

A. Filler is with the Department of Business Studies, University of Vienna, and the Department of Mathematical Methods in Economics, Technical University of Vienna, e-mail: alexander.filler@tuwien.ac.at econometric modelling, and especially in working with AR models to use a backward shift operator, which we designate by $q=z^{-1}$. This is the approach followed in this paper.

Definition 1: An autoregressive system (1) is called singular (regular) if the variance matrix $\mathbb{E}\left(\nu_{t} \nu_{t}^{\prime}\right)$ has rank $m<p$ $(m=p)$.

Clearly, $\nu_{t}$ may be written as

$$
\nu_{t}=B \varepsilon_{t}, B \in \mathbb{R}^{p \times m}
$$

where $\left(\varepsilon_{t}\right)$ is white noise with covariance matrix $\mathbb{E}\left(\varepsilon_{t} \varepsilon_{t}^{\prime}\right)=$ $2 \pi I_{m}$. The spectral density of the stationary solution of (1), $f_{y}$ is of the form

$$
f_{y}(\lambda)=W\left(e^{-i \lambda}\right) W^{*}\left(e^{-i \lambda}\right)
$$

where $W(q)=D^{-1}(q) B$ and * denotes the conjugate transpose. Clearly $f_{y}$ is rational and is singular for $m<p$.

In this paper we are concerned with singular AR systems. The importance of singular AR systems comes from the following fact [5], [6]: Let us commence from a rational and singular spectral density of rank $m<p, \forall \lambda \in[-\pi, \pi]$, and consider its $p \times m$ spectral factors $W(q)$ where $W(q)$ has no poles for $|q| \leq 1$ and is of full column rank $m$ in the same region. Then these spectral factors, which are unique up to postmultiplication by orthogonal matrices, are generically transfer functions of AR systems. This is a consequence of the fact that tall rational transfer functions are generically zeroless, i.e. have no finite zeros in the sense that $W(q)$ has rank $m$ for all $q \in \mathbb{C}$. Unless the contrary is stated explicitely here zero means a finite zero.

In generalized dynamic factor models (GDFM's) (see e.g. [7]) the latent variables have singular rational spectra. This is what has triggered our interest in singular AR systems. In more detail, the situation there is as follows (see [8]):

For the statistical analysis of GDFM's the cross-sectional dimension $p$ of $y_{t}$ is going to infinity, whereas $\varepsilon_{t}$ does not depend on $p$. Using an obvious notation we can write

$$
y_{t}^{p}=W_{p}(q) \varepsilon_{t}
$$

In addition $y_{t}^{p}$ and $W_{p}$ are assumed to be nested in the sense that for all $p$ the first rows of $W_{p+1}$ are $W_{p}$. 
For regular $\mathrm{AR}$ processes, the matrices

$$
\Gamma_{h}=\left(\begin{array}{cccc}
\gamma(0) & \gamma(1) & \ldots & \gamma(h-1) \\
\gamma(-1) & \gamma(0) & & \\
\vdots & \ddots & & \\
\gamma(-h+1) & & & \gamma(0)
\end{array}\right)
$$

are well known to be nonsingular for all $h$. On the contrary, this is not true for singular AR systems; here $\Gamma_{k+1}$ is singular and $\Gamma_{k}$ may be singular with $k$ as in (2) (see [8]); a fact which creates problems analysed in this contribution. In particular we address the following questions:

1) How are different AR models of the same spectrum related?

2) How can we construct a canonical model?

\section{Fractional Descriptions of Transfer FUNCTIONS}

For an analysis of observational equivalence, in a first step we commence from the spectral density $f_{y}$. As has been noted above, the transfer function $W(q)$ is unique only up to postmultiplication by $m \times m$ orthogonal matrices. In order to make $W(q)$ unique, we proceed as follows:

As $W(q)$ has no zeros, $W(0) \in \mathbb{R}^{p \times m}$ has full column rank $m$. The proposition below shows how a unique transfer function can be chosen:

Proposition 1: Let $A \in \mathbb{R}^{p \times m}$ with $p \geq m$ and $\operatorname{rk}(A)=$ $m$, then there exists a unique factorisation of $A=R Q$ where $Q \in \mathbb{R}^{m \times m}$ is orthogonal and $R \in \mathbb{R}^{p \times m}$ is a quasi lower triangular matrix, i.e. if the first row of $A$ is not zero then $r_{11} \neq 0$, and $r_{1 j}=0, j>1$, where $r_{i j}$ is the $(i, j)$ element of $R$, otherwise $r_{1 j}=0, j \geq 1$. If the second row of $A$ is linearly independent of the first, then $r_{22} \neq 0$ and $r_{2 j}=$ $0, j>2$ and otherwise $r_{2 j}=0, j \geq 2$ etc.

The proof is straight forward from the following observation: Note that there exists an $m \times m$ submatrix of $A$ which is regular and therefore has a unique QR description assuming $\operatorname{sign}\left(r_{i i}\right)=1$.

Thus $W(q)$ can be made unique by chosing $W(0)$ as the unique quasi lower triangular matrix $R$ from the proposition above.

Now, we commence from a unique transfer function $W(q)$. Note that the normalization described above rules out certain transfer functions, but does not restrict the equivalence classes of AR systems for a given feasible transfer function $W(q)$.

Note that in the definition of a singular AR model, there is no requirement that the polynomial matrix fraction description $D^{-1}(q) B$ be coprime. A polynomial matrix fraction representation $A^{-1}(q) B(q)$ of a transfer function matrix is said to be coprime [1], [2] if and only if $[A(q) B(q)]$ has full rank for all $q$. Nevertheless, the following result is straightforward to prove.

Theorem 1: Let a $p \times m$ transfer function

$$
W(q)=D^{-1}(q) B
$$

correspond to a singular AR model with $p>m$. Then there exists a coprime fractional description of the form

$$
W(q)=\tilde{D}^{-1}(q)\left[\begin{array}{c}
I_{m} \\
0
\end{array}\right]
$$

where $|\tilde{D}(q)|$ has all zeros in $|q|>1$.

Proof: With $W(q)$ as described in the theorem statement, observe first that without loss of generality, $B$ can be assumed to be of the form

$$
B=\left[\begin{array}{c}
I_{m} \\
0
\end{array}\right]
$$

For let $Z$ be a $p \times p$ constant nonsingular matrix such that

$$
Z B=\left[\begin{array}{c}
I_{m} \\
0
\end{array}\right]
$$

Set also $D_{1}(q)=Z D(q)$. Then we have

$$
W(q)=D_{1}^{-1}(q)\left[\begin{array}{c}
I_{m} \\
0
\end{array}\right]
$$

If this fractional description is coprime, we are done. Suppose then that it is not coprime. Now there always exist coprime fractional descriptions of $W(q)$; call one such coprime description $\bar{D}, \bar{N}$. Then we have

$$
W(q)=\bar{D}^{-1}(q) \bar{N}(q)=D_{1}^{-1}(q)\left[\begin{array}{c}
I_{m} \\
0
\end{array}\right]
$$

Because $\bar{D}, \bar{N}$ is coprime, there exists a $p \times p$ nonsingular polynomial $Q(q)$ such that

$$
\begin{aligned}
& D_{1}(q)=Q(q) \bar{D}(q) \\
& {\left[\begin{array}{c}
I_{m} \\
0
\end{array}\right]=Q(q) \bar{N}(q)}
\end{aligned}
$$

The first of these equations ensures that the zeros of $|\bar{D}(q)|$ are a subset of those of $\left|D_{1}(q)\right|$, which are the same as those of $|D(q)|$, and so all zeros of $|\bar{D}(q)|$ lie in $|q|>1$. The second of these equations ensures that $\bar{N}(q)$ has full column rank for all $q$. Hence ([1], [2]), $\bar{N}(q)$ can be expanded to a unimodular (i.e. constant nonzero determinant) polynomial $P(q)^{-1}$ such that

$$
\tilde{N}:=P \bar{N}=\left[\begin{array}{c}
I_{m} \\
0
\end{array}\right]
$$

Define also

$$
\tilde{D}:=P \bar{D}
$$

Then necesssarily, $\tilde{D}, \tilde{N}$ are coprime. Note also that because $P$ is unimodular, the zeros of $|\tilde{D}(q)|$ are the same as those of $|\bar{D}(q)|$ and therefore all lie in $|q|>1$.

Now we have four fractional representations for $W(q)$ of the form 


$$
\begin{aligned}
W(q)= & \tilde{D}(q)^{-1}\left[\begin{array}{c}
I_{m} \\
0
\end{array}\right]=\bar{D}^{-1}(q) \bar{N}(q) \\
& =D_{1}(q)^{-1}\left[\begin{array}{c}
I_{m} \\
0
\end{array}\right]=D(q)^{-1} B
\end{aligned}
$$

The first and second of these are coprime. Put another way, instead of starting with (5), we can start with a more specialized form for the coprime fraction, without any loss of generality.

We remark that although the numerator matrix of $W(q)$ has zero degree and the denominator matrix has positive degree, $W(q)$ may have a pole at infinity. Also, though $W(q)$ is zeroless, it may have a zero at $q=\infty$. Consider

$$
\begin{array}{r}
W(q)=\left[\begin{array}{cc}
1 & 1 \\
q+1 & q
\end{array}\right]^{-1}\left[\begin{array}{l}
1 \\
0
\end{array}\right]=\left[\begin{array}{c}
-q \\
q+1
\end{array}\right] \\
W(q)=\left[\begin{array}{ll}
q & 2 \\
2 & 1
\end{array}\right]^{-1}\left[\begin{array}{l}
1 \\
0
\end{array}\right]=\left[\begin{array}{c}
\frac{1}{q-4} \\
\frac{-2}{q-4}
\end{array}\right]
\end{array}
$$

The following corollary gives a description of the equivalence class of all stable singular autoregressive systems satisfying (7).

Corollary 1: Given a coprime matrix fraction description

$$
W(q)=\tilde{D}^{-1}\left[\begin{array}{c}
I_{m} \\
0
\end{array}\right]
$$

with $\tilde{D}(q)$ having all determinantal zeros in $|q|>1$, then there exists another matrix fraction description, where

$$
W(q)=\breve{D}^{-1}\left[\begin{array}{c}
I_{m} \\
0
\end{array}\right]
$$

with $\breve{D}(q)$ having all determinantal zeros in $|q|>1$, if and only if there exists a nonsingular polynomial $V(q)$ with all determinantal zeros in $|q|>1$, satisfying

$$
V(q)\left[\begin{array}{c}
I_{m} \\
0
\end{array}\right]=\left[\begin{array}{c}
I_{m} \\
0
\end{array}\right]
$$

and

$$
\breve{D}(q)=V(q) \tilde{D}(q)
$$

Moreover, this second fractional description is coprime if and only if $V(q)$ is unimodular.

Proof: The proof is a straight forward consequence of Theorem 2.2.1 in [9].

It is easy to provide more structure for the matrices $V(q)$ that arise in the above corollary.

Suppose that we partition $V(q)$ as

$$
V=\left[\begin{array}{ll}
V_{11} & V_{12} \\
V_{21} & V_{22}
\end{array}\right]
$$

where $V_{11}$ is $m \times m$. Then we necessarily have from (19)

$$
\left[\begin{array}{l}
V_{11} \\
V_{21}
\end{array}\right]=\left[\begin{array}{c}
I_{m} \\
0
\end{array}\right]
$$

and $V_{22}(q)$ has all determinantal zeros in $|q|>1$. Further, $V_{22}(q)$ is unimodular if and only if $V(q)$ is unimodular. The matrix $V_{12}(q)$ is free.

\section{CANonical Representative of EQuivalence CLASS}

The previous section has shown how to construct the equivalence class of all singular AR fractional descriptions of a transfer function matrix given one such description, irrespective of whether that description comes from a coprime or non-coprime matrix fraction. In this section, our interest is in finding a canonical form, i.e. a uniquely defined representative of the equivalence class. The equivalence class is defined by the transformation

$$
\tilde{D}=\left[\begin{array}{c}
\tilde{D}_{1} \\
\tilde{D}_{2}
\end{array}\right] \rightarrow\left[\begin{array}{c}
\tilde{D}_{1}+V_{12} \tilde{D}_{2} \\
V_{22} \tilde{D}_{2}
\end{array}\right]
$$

where $\left(\tilde{D}(q),\left(I_{m}, 0\right)^{\prime}\right)$ is coprime and $\tilde{D}_{1}$ is $m \times p$. Many questions involving polynomial matrices appearing in matrix fraction descriptions of transfer functions involve taking the matrices to be row proper or column proper, which ensures a minimization of the members of a certain set of integer variables (the row degrees or the column degrees), and then choosing a canonical member of a set of polynomial matrices restricted to the chosen set of integer parameters. We shall first consider the question: how can we choose the matrices $V_{12}, V_{22}$ to ensure that the transformed matrix, $\tilde{D}_{2}$ at least, has minimum row degrees and in addition is canonical. Then we shall focus on securing a canonical representative including some control over column degrees.

\section{A. Choice of $V_{22}$ to secure canonical $\tilde{D}_{2}$ with minimum row degrees}

We shall first consider the choice of $V_{22}$ with a view to getting a canonical form for $V_{22} \tilde{D}_{2}$. This is actually standard.

We now recall some material from [1], [2]. Consider a $r \times s$ polynomial matrix $D(q)$ with $r \leq s$ and suppose that $k_{i}$ is the degree of the $i$-th row of $D(q)$, i.e. the maximum degree of any entry of the row. The value $k_{i}=0$ means that the $i$-th row is independent of $q$, and is nonzero. By convention, the value $k_{i}=-\infty$ is used for a row with all zero entries. If there exists a square $r \times r$ submatrix of $D(q)$ whose determinantal degree is $\sum k_{i}$, the matrix is said to be row reduced. Second, a $r \times s$ polynomial matrix $X(q)$ of normal rank $r$ is said to be in Popov form or row polynomialechelon form if the following properties hold:

1) It is row reduced and the row degrees are in descending order, say $k_{1} \geq k_{2} \geq \cdots \geq k_{r}$. [It is actually conventional to assume the row degrees are in ascending order; however, the difference is immaterial, and the choice of descending order facilitates the statement of certain results later.] 
2) For row $i$ with $1 \leq i \leq r$, there is a pivot index $p_{i}$ such that $d_{i p_{i}}$ is monic and has degree $k_{i}$, and degree $d_{i j}<k_{i}$ for all $j>p_{i}$

3) if $k_{i}=k_{j}$ and $i<j$, then $p_{i}<p_{j}$, i.e. the pivot indices corresponding to the same row degree are increasing

4) $d_{i p_{j}}$ has degree less than $k_{j}$ if $i \neq j$.

Several points should be noted. First, the last two conditions imply that for all $i \neq j$, there holds $p_{i} \neq p_{j}$. Second, in the event that for some $i$, there holds $d_{i p_{i}}=1$, i.e. the $i$ th row pivot entry is 1 , then all entries to the right in the same row and all other entries in the same column are zero. Third, and we will use this fact below, the matrix formed by deleting all columns not containing a pivot index, call it $E_{2}$, will be square, will have a single entry in each column of highest degree for that column, and be column proper with highest degree coefficient matrix equal to the identity with its columns permuted.

The major results are as follows:

Lemma 1: Given an arbitrary $r \times s$ polynomial $D(q)$ with $r \leq s$ and of full row rank, there exists a unimodular left multiplying matrix such that the product is row reduced. Further, the set of row degrees is unique, i.e. if a different unimodular left multiplier produces a row reduced product, the row degrees must be the same (though not necessarily their order). In addition, every full row rank polynomial matrix can be reduced through pre-multiplication by a unimodular matrix to row polynomial-echelon form, and the form is canonical, i.e. any two full row rank polynomial matrices which are related through premultiplication by a unimodular matrix have the same row polynomial-echelon form.

This result indicates how the matrix $V_{22}$ should be chosen in (23): it should bring $\tilde{D}_{2}$ to row polynomial-echelon form.

Actually, the above analysis apparently only considers what can be done when the matrix $V_{22}$ in (23) is restricted to being unimodular. In fact, if we were to allow multiplication by any nonsingular polynomial matrix, it is readily shown that a nonunimodular multiplier will destroy the row reduced property, i.e. one or more members of the set of row degrees of $V_{22} \tilde{D}_{2}$ will be higher than members of the set of row degrees when the canonical form is chosen. There is therefore no point in considering further such transformations.

\section{B. Choice of $V_{12}$ to secure canonical $\tilde{D}_{1}$}

Observe first that the task of choosing $V_{12}$ in (23) is not affected if there is a prior choice of $V_{22}$. This is because

$$
V_{12} \tilde{D}_{2}=\left[V_{12} V_{22}^{-1}\right] V_{22} \tilde{D}_{2}
$$

and with $V_{22}$ unimodular, it is evident that $V_{12} V_{22}^{-1}$ will be polynomial if and only if $V_{12}$ is polynomial.

So we suppose that $\tilde{D}_{2}$ is in row polynomial-echelon form, and we seek to exploit the freedom in $V_{12}$ to minimize the row degrees of a certain submatrix of $\tilde{D}_{1}+V_{12} \tilde{D}_{2}$ to select a canonical representative for $\tilde{D}_{1}+V_{12} \tilde{D}_{2}$.

To obtain a canonical member of the associated equivalence class, proceed as follows. Identify the columns contain- ing pivot indices of the rows of $\tilde{D}_{2}$. With $E_{2}$ the square submatrix determined by deleting the non-pivot-index columns of $\tilde{D}_{2}$, let $E_{1}$ denote the submatrix of $\tilde{D}_{1}$ comprising the columns with the same indices. Thus $\left[\begin{array}{ll}E_{1}^{T} & E_{2}^{T}\end{array}\right]^{T}$ will be obtained from $\left[\begin{array}{ll}\tilde{D}_{1}^{T} & \tilde{D}_{2}^{T}\end{array}\right]^{T}$ by deleting columns not containing pivot indices of $\tilde{D}_{2}$. As noted above, $E_{2}$ is column proper. Form the matrix $E_{1} E_{2}^{-1}$ and express it as the sum of a polynomial term $-V_{12}$ and strictly proper remainder. This additive decomposition is of course unique. Then evidently, for some polynomial $F_{1}$, there holds

$$
E_{1} E_{2}^{-1}=-V_{12}+F_{1} E_{2}^{-1}
$$

or equivalently

$$
F_{1}=E_{1}+V_{12} E_{2}
$$

Further, because $E_{2}$ is column proper and $F_{1} E_{2}^{-1}$ is strictly proper, the column degrees of $F_{1}$ will be less than those of $E_{2}$. Now take the new $\tilde{D}_{1}$ to be precisely $\tilde{D}_{1}+V_{12} \tilde{D}_{2}$, and it is evident that in those columns of $\tilde{D}_{1}$ below which $\tilde{D}_{2}$ has a pivot index, the degree of the entries will be less than the degree of the pivot index element.

It is not hard to argue that the transformed $\tilde{D}_{1}$ is unique, i.e. we have obtained a canonical representative, because the additive decomposition referred to above is unique.

We sum up the construction as follows

Theorem 2: Consider a tall transfer function matrix $W(q)=\tilde{D}^{-1}\left[\begin{array}{ll}I_{m} & 0\end{array}\right]^{T}$ where $\tilde{D}(q)$ has all determinantal zeros in $|q|>1$ and $\left(\tilde{D}(q),\left(I_{m}, 0\right)^{\prime}\right)$ is coprime. Consider other singular AR descriptions of $W(q)$ obtained as $[V(q) \tilde{D}(q)]^{-1}\left[I_{m} 0\right]^{T}$ via a unimodular matrix satisfying

$$
V(q)=\left[\begin{array}{cc}
I_{m} & V_{12} \\
0 & V_{22}
\end{array}\right]
$$

Then a canonical representative in the set of coprime factorizations with numerator $\left[\begin{array}{ll}I_{m} & 0\end{array}\right]^{T}$ is obtainable by the following two step procedure:

1) Choose a unimodular $V_{22}$ such that $V_{22} \tilde{D}_{2}$ is in row proper echelon form

2) Let $E_{2}$ be the submatrix of (the transformed) $\tilde{D}_{2}$ comprising those columns defined by the pivot indices of $\tilde{D}_{2}$ and let $E_{1}$ be the submatrix of $\tilde{D}_{1}$ defined by selecting the same columns. Choose $V_{12}$ such that the column degrees of $F_{1}=E_{1}+V_{12} E_{2}$ are less than the corresponding column degrees of $E_{2}$.

Note that the column degrees of $F_{1}$ have been shown to be bounded by the construction above. As can be shown upper bounds for the row degrees of $\tilde{D}_{1}+V_{12} \tilde{D}_{2}$ can be obtained, where the bounds depend on $\mathrm{p}$.

\section{COUNTING THE NUMBER OF REAL PARAMETERS IN THE CANONICAL FORM}

In the normal course of events, one might expect that a canonical form for the $p \times p$ denominator matrix $\tilde{D}(q)$ of a coprime fractional description of a tall $p \times m$ transfer function matrix $W(q)$ with numerator matrix $\left[I_{m} 0\right]^{T}$ would be likely to have $O\left(p^{2}\right)$ real parameters. In this section, we shall show that, under certain conditions, the number is 
$O(p)$, other things being equal. This is a key advantage, if not requirement, of a realization of a Generalized Dynamic Factor Model.

We shall make some new assumptions in this section.

Assumptions A set of transfer functions $W_{p}(q)$ with $p=$ $m+1, m+2, \ldots$ is specified where

1) $W_{p}(q)$ is $p \times m$ for some fixed $m$ and all $p$.

2) The $W_{p}(q)$ are nested, in the sense that for all $p$ the first $p$ rows of $W_{p+1}(q)$ are $W_{p}(q)$

3) There exists some $p_{0}$ such that for all $p \geq p_{0}$, (a) the rank of $W_{p}(q)$ is $m$, (b) the McMillan degree of $W_{p}(q)$ is the same as that of $W_{p_{0}}(q)$ and (c) $W_{p_{0}}(q)$ has no zeros (apart possibly from $q=\infty$ ), and therefore the same is true of $W_{p}(q)$.

Our goal in this section is to demonstrate the existence of a parametrisation of $W_{p}(q)$ in which the number of real parameters grows only as fast as $p$; this will be done by depicting a nesting property for the denominator matrices in the canonical singular AR descriptions of the $W_{p}(q), p=$ $p_{0}, p_{0}+1, \ldots$ To do this, we need to first recall some less well known property concerning matrix fraction descriptions. The source for these is [2].

\section{A. Kronecker indices and the defect of a rational transfer function matrix}

Let $W(q)$ be a rational transfer function matrix. The Kronecker indices of $W(q)$ are closely related to its left or right null spaces. The left (right) null space $\mathcal{N}_{l}\left(\mathcal{N}_{r}\right)$ is a vector space of rational vectors in $q$ such that $f(q) W(q)=0$ $(W(q) f(q)=0)$ for any vector $f(q) \in \mathcal{N}_{l}\left(f(q) \in \mathcal{N}_{r}\right)$. It is trivial that if one of these nullspaces is nontrivial, then there exist polynomial vectors in the nullspace and indeed polynomial bases for the nullspace. Given this fact, it is then not hard to see that there exist minimal polynomial bases, i.e. ones for which the degrees of the rows (left nullspace) or columns (right nullspace) are minimal. The set of degrees arising in a minimal polynomial basis is unique. A necessary and sufficient condition on the polynomial matrix whose rows (columns) make up the nullspace for those rows (columns) to form a minimal basis is that it be row (column) proper and with no nontrivial left (right) greatest common divisor, or equivalently with full row (column) rank for all finite $q$.

Definition 2: Suppose that the nontrivial left (right) null space of $W(q)$ exists and has a minimal polynomial basis, $f_{1}(q), \cdots, f_{\gamma}(q)$, whose degrees are $\mu_{1}, \cdots, \mu_{\gamma}$ with $\mu_{1} \geq$ $\cdots \geq \mu_{\gamma}$. Then $\mu_{1}, \cdots, \mu_{\gamma}$ are called the left (right) Kronecker indices.

The next concept is that of the defect of a rational matrix $W(q)$. This can be defined using properties of the SmithMcMillan form of $W(q)$. However, we shall take as our starting point the following:

Definition 3: Let $n$ and $n_{z}$ be the McMillan degree and the number of zeros, (allowing for zeros at $q=\infty$ ), of $W(q)$. Then the defect of $W(q)$ is defined as

$$
\operatorname{def} W(q)=n-n_{z}
$$

The defect is related to the Kronecker indices, Theorem 6.511 of [2].The result is as follows:

Theorem 3: Let $W(q)$ be a rational transfer function matrix. Then def $W(q)$ is the sum of the left and right Kronecker indices of $W(q)$.

\section{B. Structure of the canonical denominator matrices}

We will now use the result on the defect of a rational matrix to determine more aspects of the structure of a canonical singular AR description.

Adopt the assumptions at the start of the section, and fix some $p \geq p_{0}$. Let

$$
W_{p}(q)=\tilde{D}_{p}^{-1}\left[\begin{array}{c}
I_{m} \\
0
\end{array}\right]=\left[\begin{array}{c}
\tilde{D}_{1 p} \\
\tilde{D}_{2 p}
\end{array}\right]^{-1}\left[\begin{array}{c}
I_{m} \\
0
\end{array}\right]
$$

where the denominator matrix is canonical, $\tilde{D}_{1 p}$ is $m \times p$, $\tilde{D}_{2 p}$ is $(p-m) \times p$, and is in row polynomial-echelon form. Then we claim:

Lemma 2: The left Kronecker indices of $W_{p}(q)$ are precisely the row degrees of $\tilde{D}_{2 p}$.

Proof: From (29), it is immediate that $\tilde{D}_{p} W(q)=$ $\left[\begin{array}{ll}I_{m} & 0\end{array}\right]^{T}$, from which one has

$$
\tilde{D}_{2 p} W(q)=0
$$

Notice that $W(q)$, being of size $p \times m$, has a left nullspace of dimension $p-m$, which is the number of rows of $\tilde{D}_{2 p}$; further this last matrix is polynomial and row reduced. Further, because the matrix fraction $\tilde{D}_{p}^{-1}\left[\begin{array}{ll}I_{m} & 0\end{array}\right]^{T}$ is coprime, the following matrix has full row rank for all finite $q$ :

$$
G(q)=\left[\begin{array}{cc}
\tilde{D}_{1 p}(q) & I_{m} \\
\tilde{D}_{2 p}(q) & 0
\end{array}\right]
$$

from which it is trivial that $\tilde{D}_{2 p}(q)$ has full row rank for all finite $q$. Together, the full rank property and the row reduced property imply that the row degrees of $\tilde{D}_{2 p}$ are precisely the left Kronecker indices of $W(q)$.

With this Lemma in hand, we can state the main result of this section.

Theorem 4: Adopt the assumptions listed at the start of the section, and let $n$ denote the McMillan degree of $W_{p_{0}}(q)$. Then there exists an integer $p_{1}$ with $m+n \geq p_{1} \geq p_{0}$ such that for all $p>p_{1}$, there holds

$$
\tilde{D}_{p}=\left[\begin{array}{c}
\tilde{D}_{1 p} \\
\tilde{D}_{2 p}
\end{array}\right]=\left[\begin{array}{cc}
F_{11}(q) & 0 \\
F_{21}(q) & 0 \\
F_{31} & I
\end{array}\right]
$$

where $\tilde{D}_{1 p}$ is $m \times p, F_{11}(q)$ is polynomial and $m \times p_{1}$, $F_{21}(q)$ is polynomial and $\left(p_{1}-m\right) \times p_{1}, F_{31}$ is constant and $\left(p-p_{1}\right) \times p_{1}$ and the identity matrix is $\left(p-p_{1}\right) \times\left(p-p_{1}\right)$. Moreover, for $p>p_{1}$, the $\tilde{D}_{p}$ are nested, in that $\tilde{D}_{p-1}$ is obtainable from $\tilde{D}_{p}$ by deleting the last row and column. 
Proof: We shall first identify the structure of $\tilde{D}_{2 p}$. Recall that the assumptions on $W_{p}(q)$ ensure that it has no zeros other than possibly at $q=\infty$ (and suppose there are $n_{p \infty}$ such zeros), and its McMillan degree is the same for all $p \geq$ $p_{0}$, say $n$. Further, it has full column rank, and thus no right Kronecker indices. Then by Theorem 3 and the preceding lemma the sum of the left Kronecker indices, which is the sum of the row degrees of $\tilde{D}_{2 p}$, is $n-n_{p \infty} \leq n$, and so the number of rows of $\tilde{D}_{2 p}$ with positive row degree is bounded by $n$. Then because the row degrees are decreasing, there is a $p_{1} \geq p_{0}$ with $p_{1} \leq m+n$ such that for any $i>p_{1}$ and any $p>p_{1}$, the row degrees are all zero. (Notice that no row degree can be $-\infty$, since $\tilde{D}_{p}$ is necessarily a nonsingular matrix). Recall further that all entries to the right of, above and below a pivot element equal to 1 must be zero. All this means that for $p \geq p_{1}$, the matrix $\tilde{D}_{2 p}$ has the following structure:

$$
\tilde{D}_{2 p}=\left[\begin{array}{cc}
F_{21}(q) & 0 \\
F_{31} & I
\end{array}\right]
$$

where $F_{21}(q)$ is $\left(p_{1}-m\right) \times p_{1}$ and is in row polynomialechelon form, and $F_{31}$ is $\left(p-p_{1}\right) \times p_{1}$, this following from the fact that the last $p-p_{1}$ rows of $\tilde{D}_{2 p}$ have row degree zero, and are part of a matrix in row polynomial-echelon form. The identity matrix is of course $\left(p-p_{1}\right) \times\left(p-p_{1}\right)$.

The structure of $\tilde{D}_{1 p}=\left[F_{11}(q) 0\right]$, and in particular the fact that the last $\left(p-p_{1}\right)$ columns are zero, is a consequence of the canonical form construction, which ensures that in those columns of $\tilde{D}_{1 p}$ which correspond to pivot indices appearing in $\tilde{D}_{2 p}$, the degree of each entry is less than that of the corresponding pivot entry. The nonzero entries of the identity matrix in the bottom right corner of $\tilde{D}_{2 p}$ are all pivot entries, and this gives rise to the zeros in the last $\left(p-p_{1}\right)$ columns of $\tilde{D}_{1 p}$.

Last, to establish the nesting property, recognize that $W_{p-1}(q)=\left[\begin{array}{ll}I_{p-1} & 0\end{array}\right] W_{p}(q)$, where the submatrix of zeros is in fact a $(p-1)$-dimensional vector of zeros. Denote the matrix obtained from $\tilde{D}_{p}$ by deleting the last row and column as $\hat{D}_{p-1}$. It is easily verified that

$$
\tilde{D}_{p}^{-1}=\left[\begin{array}{cc}
\hat{D}_{p-1}^{-1} & 0 \\
X & 1
\end{array}\right]
$$

where $X$ is a row vector whose entries are inessential. It follows that

$W_{p-1}(q)=\left[\begin{array}{ll}I_{p-1} & 0\end{array}\right]\left[\begin{array}{cc}\hat{D}_{p-1}^{-1} & 0 \\ X & 1\end{array}\right]\left[\begin{array}{c}I_{m} \\ 0\end{array}\right]=\hat{D}_{p-1}^{-1}\left[\begin{array}{c}I_{m} \\ 0 \\ (35)\end{array}\right]$

It is trivial to check that $\hat{D}_{p-1}$ is canonical because $\tilde{D}_{p}$ has this property. Hence there necessarily holds $\hat{D}_{p-1}=\tilde{D}_{p-1}$, as required.

\section{Counting parameters}

Using Theorem 4 and with the assumptions on the sequence $W_{p}(q)$ as stated at the beginning of the section, it is easy to show that the number of real parameters required in the singular AR description of $W_{p}(q)$, i.e. the number of real parameters in $\tilde{D}_{p}$ (excluding any necessarily taking the value 0 or 1 ), grows linearly with $p$. For $p>p_{1}$, extra parameters arise when changing $p$ through the addition of further rows to $F_{31}$, as in equation (32). Increase of $p$ by 1 increases the number of real parameters by the number of columns in $F_{31}$, which is $p_{1}$. Since $p_{1}$ is independent of $p$, this demonstrates the linear relationship between $p$ and the number of real parameters.

\section{CONCLuSions}

In this paper, we have considered tall transfer function matrices of dimension $p \times m$ which have no finite zeros. Such transfer functions can be represented as singular AR systems. Observational equivalence and canonical forms are discussed. When $p$ varies with the associated transfer function matrices remaining nested and of bounded McMillan degree, a nesting property for the singular AR descriptions can be found, and the total number of real parameters in the representation grows linearly with the number of rows of the transfer function.

Our result has been motivated by the analysis of Generalized Dynamic Factor Models which are used for modeling and forecasting for high dimensional time series. The following problems are left for further research

1) The integer valued parameters $k_{1}, \ldots, k_{p-m}$ and $p_{1}, \ldots, p_{p-m}$ associated with our canoncial form do not directly describe the set of all canonical forms associated with the same integers. This is a consequence of the fact that we have neither been able to give exact bounds of the degree of those columns of $D_{1}$ not corresponding to pivote indices, nor that the corresponding real valued parameters are free.

2) We have not discussed the question whether the canonical form proposed corresponds to a $D$ of minimal degree $k$ in (2).

3) The structure theory in this paper has been developed in view of the more general problem of identification in particular of GDFM's. Major open questions are estimation of integer and real valued parameters.

\section{REFERENCES}

[1] W. Wolovich: Linear Multivariable Systems, Springer, New York, 1974

[2] T. Kailath: Linear Systems, Prentice Hall, Englewood Cliffs, N.J., 1980

[3] C. C. MacDuffee: The Theory of Matrices, Chelsea, New York, 1950

[4] B.D.O. Anderson, M. Deistler: Properties of Zero-free spectral matrices, forthcoming in IEEE Tr.AC

[5] B.D.O. Anderson, M. Deistler: Properties of Zero-free Transfer Function Matrices, SICE Journal of Control, Measurement and Systemintegration, Vol. 1, No. 4, 284 - 292, 2008

[6] B.D.O. Anderson, M. Deistler: Generalized Linear Dynamic Factor Models A Structure Theory, CDC Cancun, 2008

[7] M. Forni, M. Lippi: The generalized dynamic factor model: representation theory, Econometric Theory, 2001

[8] A. Filler, M. Deistler, B.D.O. Anderson, Ch. Zinner, W. Chen: Generalized Linear Dynamic Factor Models - An Approach via Singular Autoregressions, ECC, 2009

[9] E. J. Hannan, M. Deistler: The Statistical Theory of Linear Systems, Wiley, New York, 1988 